\title{
Impacto do tratamento ortodôntico nos tecidos periodontais: uma revisão narrativa da literatura multidisciplinar.
}

Jean Franco Mendes Sá (1) e Saulo Manuel Ferreira (1)

\section{ARTIGO DE REVISÃO}

Resumo

O objetivo desta revisão é descrever as alterações mais comumente observadas no periodonto causadas pelo tratamento ortodôntico, a fim de facilitar a colaboração e comunicação dos especialistas. Uma busca em banco de dados eletrônico foi realizada usando o banco de dados de resumos e citações do PubMed e material bibliográfico foi usado para encontrar outras fontes apropriadas. As alterações dos tecidos periodontais moles e duros durante o tratamento ortodôntico e a manutenção dos pacientes são discutidas a fim de fornecer um quadro exaustivo das possíveis interações entre essas duas disciplinas entrelaçadas.

Palavras-chave: Tratamento ortodôntico; Tecidos periodontais; Revisão de literatura. 


\title{
Impact of orthodontic treatment on periodontal tissues: a narrative review of the multidisciplinary literature.
}

\begin{abstract}
The purpose of this review is to describe the most commonly observed changes in the periodontium caused by orthodontic treatment, in order to facilitate collaboration and communication by experts. An electronic database search was performed using the PubMed abstract and citation database and bibliographic material was used to find other appropriate sources. Periodontal soft and hard tissue changes during orthodontic treatment and patient care are discussed in order to provide an exhaustive picture of the possible interactions between these two intertwined disciplines.
\end{abstract}

Keywords: Orthodontic treatment; Periodontal tissues; Literature review.

Instituição afiliada: 1- Grupo de pesquisa em odontologia clínica, Goiania, Goiais, Brasil. Dados da publicação: Artigo recebido em 10 de junho, revisado em 15 de junho, aceito para publicação em 20 de junho e publicado em 30 de junho.

DOI: https://doi.org/10.36557/2674-8169.2021v3n6p49-71

Jean FrancoMendez Sá dr.sa38@gmail.com

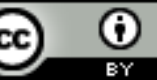

This work is licensed under a Creative Commons Attribution 4.0 International

License. 


\section{INTRODUÇÃO}

Graças à crescente demanda por aparência, o tratamento ortodôntico está sendo cada vez mais adotado na população adulta. Como os pacientes ortodônticos adultos também podem ter necessidades restauradoras e periodontais, a interação entre as diferentes especialidades torna-se ainda mais importante. Muitos pacientes periodontais podem apresentar migração dentária patológica ou outras deformidades em que a ortodontia pode representar uma parte importante do tratamento. Tanto o periodontista quanto o ortodontista devem entender os resultados do trabalho de um no outro e cooperar na prática clínica para oferecer o melhor tratamento possível aos seus pacientes.

O número de publicações avaliando a ortodontia e as interações periodontais é cada vez maior (Figura 1 ). O número de artigos publicados nos últimos 5 anos é igual ao dos publicados nos 10 anos anteriores (2000-2010), que, por sua vez, quase se iguala ao dos 60 anos anteriores (1940-2000).

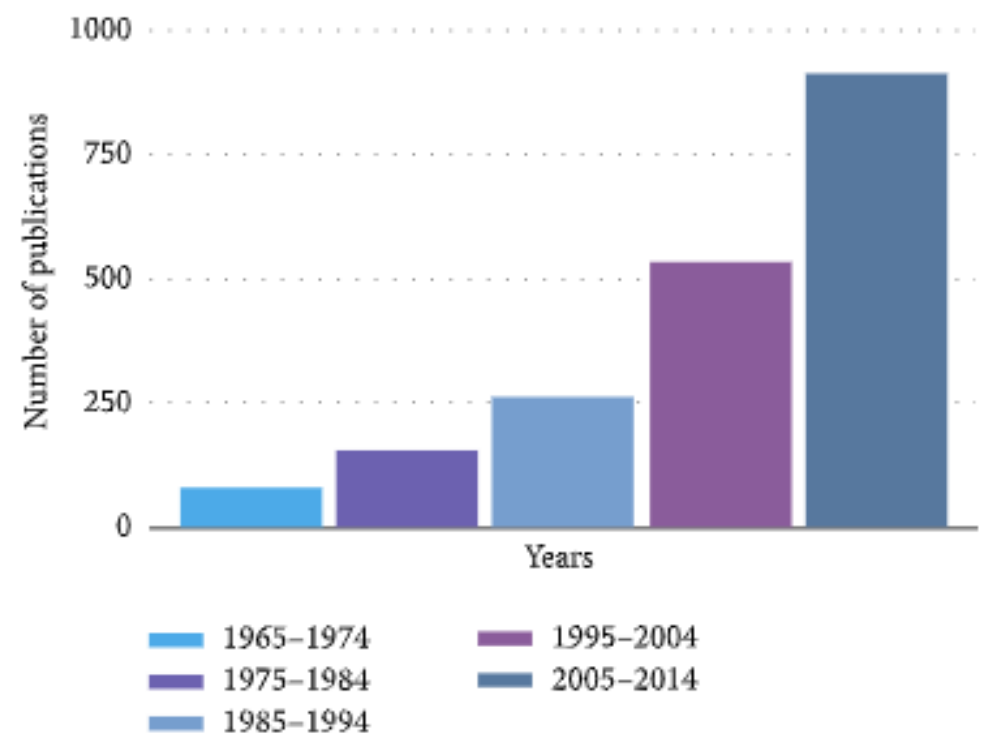

figura 1: O aumento do número de dados observando a Ortodontia com Periodontia reflete o crescente interesse na abordagem multidisciplinar com o tempo.

O objetivo desta revisão é explorar este vasto corpo de literatura, selecionar conceitos críticos específicos e conexões multidisciplinares e destacar a importância da cooperação de especialidades.

Uma busca em um banco de dados eletrônico foi realizado usando resumos e citações no PubMed com as palavras-chave: "periodontology" AND "orthodontics" publicado em inglês. Avaliações, ensaios clínicos, estudos com animais, estudos 
Impacto do tratamento ortodôntico nos tecidos periodontais: uma revisão narrativa da literatura multidisciplinar

Sá e Ferreira

comparativos, estudos de avaliação e relatos de casos foram selecionados. Duas autoras, Angelina Gorbunkova e Anna Brizhak, selecionaram os artigos. O material bibliográfico dos jornais foi então usado para encontrar outras fontes apropriadas.

Serão descritas as observações das alterações dos tecidos periodontais moles e duros durante a movimentação dentária ortodôntica (OTM) na literatura ortodôntica e periodontal.

\section{Alterações de tecido mole}

O tratamento ortodôntico pode ser implementado para melhorar a estética dentária, não apenas corrigindo a posição dos maxilares e deformidades da dentição, mas também criando as condições para melhorar a saúde gengival. Pacientes adultos previamente afetados por doença periodontal frequentemente apresentam "triângulos pretos" devido à perda da altura das papilas interdentais. Por meio da Ortodontia, é possível corrigir a posição dos dentes e melhorar a estética dos tecidos moles. Foi sugerido que a aproximação dos dentes ortodônticos pode alterar a topografia do nível da crista alveolar interproximal e melhorar a posição da papila interdental [ 1 ], embora triângulos pretos também possam aparecer como consequência do alinhamento dos dentes ao resolver o apinhamento. Tarnow et al. em 1992 [ 2 ] e Wu et al. em 2003 [3 ] sugeriram que o preenchimento do espaço interdental com a papila poderia ser determinado pela posição do ponto de contato em relação à posição da crista óssea. A remodelagem dentária pode ajudar a movimentar o ponto de contato mais apicalmente durante a aproximação ortodôntica dos dentes, o que pode ajudar a alcançar bons resultados estéticos na área interdental [ 4 ].

No entanto, deve-se levar em consideração que durante o OTM alguns efeitos adversos no tecido periodontal mole podem ser observados. As alterações que ocorrem com mais frequência nos tecidos moles são supercrescimento gengival (GO), recessões gengivais (RG) e invaginações gengivais (GIs), que comumente ocorrem em casos de extração ortodôntica.

O supercrescimento gengival é uma condição muito comum na população ortodôntica, caracterizada pelo aumento gengival, possivelmente resultando em pseudo-embolsamento com ou sem perda de inserção. Ao envolver a região anterior, pode impactar na qualidade de vida relacionada à saúde bucal [ 5 ]. Tradicionalmente, o GO era considerado uma reação inflamatória consecutiva ao acúmulo de placa bacteriana [ 6 ]. Outros fatores como irritação química produzida por materiais usados para bandagem, irritação mecânica por bandas e impactação de alimentos têm sido sugeridos para explicar a patogênese do GO [ 7 ]. Em 1972, S. Zachrisson e BU Zachrisson [ 8] relataram aumento gengival em pacientes que mantinham excelente higiene oral. Mais recentemente, Şurlin et al. [ 9] avaliaram pacientes ortodônticos com boa higiene dental exibindo GO sem quaisquer sinais clínicos de inflamação gengival. Esses pacientes 
exibiram níveis elevados de metaloproteinase-8 (MMP-8) e metaloproteinase-9 (MMP9) da matriz no líquido gengival crevicular (GCF). Considerou-se que, durante o tratamento ortodôntico, o estresse mecânico pareceu ser um dos principais fatores determinantes do aumento da produção de MMP-9 e do início do GO. Alguns autores também avaliaram o possível papel de uma reação alérgica ao níquel, liberada dos aparelhos ortodônticos de aço inoxidável. Estudos in vitro e in vivo sugerem que íons de níquel liberados podem causar uma reação alérgica dependente do tempo de exposição, caracterizada por uma proliferação de queratinócitos regulada positivamente e aumento da proliferação de células epiteliais [ 10, 11 ]. Portanto, pode ser importante tratar pacientes com aparelhos sem níquel e adotar questionários para avaliar história prévia de alergia a metais, uma vez que foram associadas a um aumento na frequência de GO [ 12 - 14 ].

O aumento das papilas interdentais e o acúmulo de tecido gengival podem surgir devido à aplicação de forças compressivas ou de retração no local de fechamento do espaço de extração. No tratamento ortodôntico, a extração de dentes, mais comumente, primeiros ou segundos pré-molares, pode ser necessária. O fechamento ortodôntico dos locais de extração pode resultar em invaginação gengival ou acúmulo de tecido gengival [ 15 ].

O crescimento gengival foi definido por Robertson et al. [ 16 ] como uma invaginação linear do tecido interproximal com orientação mesial e distal e uma profundidade de sondagem intragengival de pelo menos $1 \mathrm{~mm}$. A frequência de $\mathrm{Gl}$ é relatada como alta e pode ser observada com mais frequência na mandíbula [ 16 - 18 ]. Devido à sua localização, o Gl pode dificultar o controle adequado da placa, possivelmente contribuindo para a ocorrência de doenças gengivais e periodontais [ 16 , 19 ]. Existe uma correlação entre a fenda gengival e o tempo de OTM. Significativamente mais IGs foram relatados quando houve um atraso no fechamento do espaço e o tratamento ortodôntico foi iniciado tarde após a extração do dente [ 17 , 20]; portanto, a comunicação adequada entre especialistas é particularmente importante. O crescimento gengival pode exibir um alto grau de variabilidade, variando de uma pequena prega superficial na gengiva a defeitos graves com penetração completa da crista alveolar (25\% de todas as fissuras) [ 17 ]. De acordo com a gravidade $\mathrm{Gl}$, as estratégias de tratamento podem variar. Quando o Gl está localizado apenas em tecidos moles, pode ser tratado com lâmina fria ou eletrocautério, sem diferença significativa entre as duas técnicas de gengivectomia [ 21 ]. O laser de diodo de tecidos moles no manejo de problemas mucogengivais pode apresentar algumas vantagens devido à mínima dor pós-operatória reposta com o uso desses dispositivos [22] Para prevenir a formação de GI durante OTM na área pós-extração, a regeneração óssea guiada (GBR) pode ser aplicada; entretanto, o melhor momento para a aproximação do dente a ser iniciada após a cirurgia ainda está em discussão [ 23 - 25 ]. 


\section{Impacto do tratamento ortodôntico nos tecidos periodontais: uma revisão narrativa da literatura multidisciplinar \\ Sá e Ferreira}

Tanto a literatura ortodôntica quanto a periodontal têm discutido exaustivamente a recessão gengival que pode levar a uma estética insatisfatória, sensibilidade radicular, maior suscetibilidade a cáries, abrasão dentária e, consequentemente, dificuldades na manutenção da higiene oral. OTM pode promover a formação de GR ou melhorar as condições dos tecidos moles [ 26 - 32 ]. Entre os pacientes ortodônticos, até 10-12\% exibiram recessões gengivais [ 26,33 ]. Acredita-se que uma das principais razões para o desenvolvimento de RG seja um trauma mecânico contínuo por escova de dentes [ 34 , 35 ], mas Matthews [ 36 ] e Rajapakse et al. [ 37] sugeriram que não há boas evidências de ligação direta entre a escovação dentária e o aparecimento de RG não inflamatório. Várias características anatômicas e morfológicas foram sugeridas para desempenhar um papel na formação de GR. Durante o OTM, deiscências ósseas alveolares podem ocorrer quando as raízes dos dentes se movem através do osso cortical alveolar [ 38 - 40 ]. Mais frequentemente, este tipo de movimento é realizado em pacientes com um processo alveolar pequeno, placas ósseas vestibulares ou linguais delgadas, posição excêntrica dos dentes, seio maxilar basalmente estendido e perda óssea alveolar progressiva [ 41 ]. Deve-se notar que se o dente for movido dentro do envelope do osso alveolar, o risco de efeito colateral prejudicial no tecido mole marginal é mínimo [ 40 , 41] A direção das forças ortodônticas aplicadas também pode ter um impacto sobre os tecidos moles. Alguns estudos sugeriram que a proclinação controlada dos incisivos inferiores pode ser realizada em pacientes ortodônticos sem risco de ruptura periodontal se um bom nível de higiene dental for fornecido [ $27,33,42,43$ ]. Estudos recentes sugeriram que [ 44,450 movimento ortodôntico de pró-inclinação pode estar significativamente associado à redução da largura do tecido queratinizado. Esses achados são corroborados por outros estudos anteriores, sugerindo que o movimento dentário labial pode resultar na diminuição da espessura do tecido vestíbulo-lingual e reduzir a altura da gengiva livre facilitando a RG. Por outro lado, o movimento dentário lingual pode ter o efeito oposto [ 29 , 38, 44 ]. O biótipo periodontal também foi sugerido como um fator importante no desenvolvimento de RG. Uma forte correlação foi encontrada entre o biótipo delgado e o movimento ortodôntico de pró-inclinação em termos de profundidade do RG e largura do tecido queratinizado. Em contraste com os pacientes que realizaram um biótipo gengival espesso, aqueles com um biótipo recortado fino são considerados em risco [44 , 45 ]. O biótipo periodontal fino e a quantidade de gengiva inserida foram significativamente relacionados à espessura da placa labial e à posição da crista alveolar. O periodonto fino demonstra diminuição da resistência ao estresse mecânico ou inflamação e pode se correlacionar com o desenvolvimento de GR [ 28 , 42, 45 , 46 ]. Diante disso, uma avaliação precisa da espessura gengival antes de iniciar o OTM é definitivamente recomendada [ 44,45 ].

Como para qualquer condição com etiologia multifatorial, é importante ponderar a importância de qualquer fator contribuinte para avaliar a predisposição do paciente antes de iniciar a terapia. Por isso, recomendamos uma avaliação crítica de 
Impacto do tratamento ortodôntico nos tecidos periodontais: uma revisão narrativa da literatura multidisciplinar

Sá e Ferreira

cada caso específico antes de chegar a um plano de tratamento definitivo. Fatores relacionados ao paciente também podem desempenhar um papel importante no processo de tomada de decisão.

Enquanto aguardamos mais informações baseadas em evidências sobre como proceder em diferentes cenários de casos, gostaríamos de fornecer nossa opinião pessoal a fim de destacar áreas de interesse para possíveis pesquisas futuras.

A cirurgia mucogengival durante o tratamento ortodôntico visa alterar as características dos tecidos moles a fim de criar condições mais favoráveis para a resistência mecânica ao estresse. No entanto, as características gengivais melhoradas podem não garantir a ausência de recessão gengival após TO, especialmente quando uma expansão significativa da arcada dentária ou proclinação labial é realizada e uma segunda cirurgia pode ser necessária após o término da ortodontia.

Nosso insight ao avaliar os casos ortodônticos em risco de possível RG é que os pacientes com biótipo fino devem receber enxerto de tecido mole antes do OTM, a fim de reduzir o risco e a extensão do possível RG. Até o momento, não está claro quais características gengivais e de movimento podem predispor ao RG e qual seria a incidência de RG em cada cenário específico. A eficácia das cirurgias preventivas também deve ser analisada mais detalhadamente: por exemplo, gostaríamos de saber o número de cirurgias preventivas em correspondência com o número de pacientes que realmente desenvolveriam RG. Também gostaríamos de saber quantos pacientes que recebem uma cirurgia preventiva também precisarão de um segundo procedimento cirúrgico corretivo.

Um cenário diferente pode ser encontrado, caso o GR ocorra durante o OTM. Nestes casos, a enxertia de partes moles é indicada e deve ser realizada o mais rápido possível, pois todos os demais parâmetros (inflamação gengival, trauma, etc.) estão controlados. O objetivo é tratar a recessão, uma vez que ainda é mínima, e melhorar o prognóstico do tratamento. A terapia ortodôntica deve ser avaliada cuidadosamente neste período de tempo para determinar se deve parar ou desacelerar o OTM até que a cicatrização da ferida esteja completa. Claramente, o momento do aparecimento do RG é importante e devemos entender melhor as implicações de um RG ocorrer no terço inicial do tratamento ortodôntico versus próximo ao final do OTM.

Quando RGs preexistentes são encontrados antes do tratamento ortodôntico, o impacto do tratamento ortodôntico deve ser avaliado cuidadosamente. Se o dente for planejado para movimentação dentária lingual, a cirurgia mucogengival pode nem ser necessária e o OTM sozinho pode acabar tratando ou pelo menos não agravar a recessão. Quando necessário, o prognóstico da cirurgia mucogengival pode ser melhorado após a movimentação do dente para a língua. Caso o dente seja movido 
labialmente, um procedimento corretivo mucogengival com o objetivo de evitar a progressão da doença deve ser levado em consideração. OTM pode ser iniciado assim que a cicatrização da ferida estiver completa (3-4 meses). Ao final da terapia ortodôntica, o local deve ser reavaliado e uma segunda intervenção pode ser necessária em casos limitados.

Cada caso clínico pode incluir uma combinação de diferentes fatores predisponentes e precipitantes que podem afetar o resultado do tratamento; portanto, é importante avaliar os fatores de risco durante o planejamento do tratamento ortodôntico, a fim de evitar consequências indesejáveis da terapia administrada. 0 gerenciamento de risco é possivelmente o fator mais importante no planejamento do tratamento desses pacientes. Nós encorajamos os pesquisadores a avaliar ainda mais aspectos obscuros, como a predisposição do paciente / dente / local à recessão gengival e tipo e momento ideal de tratamento e gerar sistemas incrementais para agrupamento hierárquico, que seriam capazes de reunir diferentes nós probabilísticos na determinação de soluções clínicas.

\section{Alterações ósseas}

A força mecânica durante o OTM resulta na reabsorção e aposição óssea amplamente discutidas na literatura ortodôntica e periodontal. Na saúde, durante o OTM, todos os componentes do aparelho de fixação periodontal, incluindo a estrutura óssea, o ligamento periodontal e os componentes do tecido mole, se movem junto com o dente. O mesmo se aplica a pacientes com tecidos periodontais reduzidos, mas saudáveis [ $32,47,48$ ]. Após o tratamento periodontal, forças ortodônticas leves combinadas com um bom controle de higiene dental podem ser suficientes para resultar no alinhamento dos dentes quando o suporte periodontal é reduzido.

OTM na presença de bolsas intra-ósseas apresenta um desafio diferente para os médicos. Vários estudos sugeriram que o OTM após o tratamento periodontal cirúrgico pode ter um impacto na morfologia dos defeitos ósseos, diminuir a profundidade da bolsa e aumentar a cicatrização do tecido conjuntivo. Todas as mudanças positivas no aparelho de suporte foram alcançadas somente quando um bom controle de higiene dental foi implementado. Alguns autores aplicaram forças ortodônticas intrusivas e relataram melhorias clínicas e radiológicas [ 49,50 ]. Além disso, foi relatado no estudo histológico de Melsen et al. [ 51 ] que a nova formação de cemento e nova fixação de colágeno podem ser obtidas por intrusão ortodôntica na presença de uma boa higiene dental. da Silva et al. [52] em seu estudo em cães intrudiram dentes com defeitos de furca e sugeriram que defeitos de classe III podem ser clinicamente eliminados ou reduzidos, resultando em ganho de nível de inserção clínico. Outro estudo investigou a influência dos movimentos de inclinação na presença de bolsas intra-ósseas e reforçou a conclusão de que o OTM pode ser realizado em dentes com defeitos ósseos sem dano ao nível de inserção periodontal [ 53 ]. Polson et al. [47] avaliaram ainda mais o aparato 
de fixação em tais dentes e relataram a presença de epitélio juncional longo entre o osso e a superfície da raiz após o movimento dos dentes para dentro e através do defeito, sugerindo que não houve regeneração do aparato de suporte. Portanto, foi recomendado aplicar técnicas de GTR no tratamento de defeitos intra-ósseos antes da terapia ortodôntica, a fim de alcançar a regeneração ao invés do reparo.

A eficácia da regeneração periodontal no tratamento de defeitos intra-ósseos está bem documentada e apoiada por estudos histológicos. Todos os benefícios da regeneração tecidual guiada (GTR) podem ser mantidos por um longo período de tempo (mais de 10 anos) [ 54, 55 ]. Acredita-se comumente que a topografia de bolsa óssea é importante para o prognóstico do tratamento regenerativo; no entanto, uma revisão sistemática recente afirmou que os resultados clínicos da regeneração periodontal são influenciados pelo comportamento do paciente e pela abordagem cirúrgica mais do que pelas características do dente e do defeito [ 56] A adoção combinada de terapia ortodôntica e regeneração periodontal de dentes com defeitos infra-ósseos pode ser sugerida em múltiplas situações. Extrusão ortodôntica, intrusão e movimentos dentais sagitais com tempos diferentes de OTM após GTR foram descritos na literatura. Avaliando o crescimento apical do epitélio juncional, Nemcovsky et al. [ 57 ] sugeriram que a regeneração periodontal pode ser indicada antes do OTM. Em 2003, Diedrich et al. [ 58] realizaram um estudo sobre a intrusão ortodôntica e translação de dentes com defeitos ósseos de 3 paredes previamente tratados com desbridamento de retalho aberto combinado com proteína da matriz do esmalte. No grupo de intrusão, um ligeiro crescimento epitelial para baixo, extensa cementogênese e aposição óssea foram documentados levando a resultados comparáveis aos observados no local de tensão do grupo de translação. Defeitos no lado da pressão foram adicionalmente cobertos com membrana reabsorvível e após OTM mostraram aposição óssea marcadamente reduzida. Esses resultados podem indicar a possível influência da degradação do biomaterial nos resultados regenerativos, o que também foi sugerido em outros estudos [ 52, 59 ]. Araújo et al. [ 60] sugeriu que era possível mover os dentes para áreas previamente aumentadas com biomaterial. Forças ortodônticas foram aplicadas 3 meses após a enxertia e nenhum impedimento foi observado no OTM. Alguns autores sugeriram que o momento ideal para começar OTM após GTR é 4 semanas após a cirurgia, quando a atividade mitótica das células periodontais está aumentada e OTM ocorre em osso imaturo [ 52,61 ]. Attia et al. [ 62,63], avaliando a eficácia de diferentes tempos para o início do tratamento ortodôntico ativo após a GTR, sugeriram que melhorias significativas na regeneração periodontal podem ser observadas em defeitos tratados com a aplicação imediata de forças ortodônticas após a cirurgia. Outros demonstraram que o tratamento ortodôntico fornecido 1 ano após a GTR, quando os tecidos duros e moles estão maduros, não causou nenhum efeito prejudicial nos resultados da regeneração periodontal [ 4] Levando em consideração os resultados desses diferentes estudos, pode-se concluir que vários fatores, como direção do movimento dentário e tempo e escolha dos biomateriais, devem ser levados em 
consideração durante o planejamento do tratamento, embora sejam necessários ensaios clínicos mais bem elaborados para esclarecer melhor os mecanismos envolvidos na cicatrização de feridas quando as forças ortodônticas são aplicadas [ 64 ].

\section{Manutenção}

Nossa pesquisa inicial incluiu uma grande quantidade de estudos avaliando os efeitos de diferentes níveis de higiene oral em pacientes submetidos a OTM. Isso destaca a importância da manutenção na prática odontológica, principalmente nos casos em que a ortodontia é combinada com o tratamento periodontal. Para todos os pacientes em tratamento ortodôntico com aparelhos fixos ou com contenções fixas, é difícil manter um bom nível de higiene oral, pois as construções e acessórios ortodônticos podem dificultar a escovação e o uso do fio dental convencionais. Enquanto isso, a higiene oral deficiente em pacientes ortodônticos parece ser um fator chave no desenvolvimento de lesões de manchas brancas, cárie dentária e inflamação gengival devido à presença de acúmulo de placa dentária [ 65,66$]$ Na presença de higiene dental insuficiente, o tratamento ortodôntico pode levar à transposição da placa dentária supragengival subgengivalmente, resultando na formação de bolsa infrabônica [67 ].

O tipo de aparelho (fixo ou removível), o material do braquete, a técnica de colagem (lingual ou bucal) e o tipo de contenção selecionado para terapia ortodôntica podem influenciar a capacidade do paciente de manter um bom nível de controle da placa. Durante o mau hálito oral OTM, o índice de placa e o índice gengival aumentam e as primeiras alterações podem ser observadas imediatamente após a colagem [ 66,68 ].

Alguns autores sugeriram que o acúmulo de placa dentária em pacientes que usam aparelhos fixos é maior do que em pacientes que usam aparelhos removíveis [ 69 ]. Embora a evidência que apóie esta frase não seja a de que ensaios clínicos mais fortes e bem planejados devam ser realizados a fim de investigar os parâmetros clínicos do estado periodontal em duas modalidades de tratamento diferentes, os médicos podem querer considerar esta informação ao planejar o tratamento de pacientes periodontais para terapia ortodôntica. Os aparelhos ortodônticos linguais mostraram maior retenção de placa em comparação aos aparelhos ortodônticos labiais devido ao acesso mais difícil para manutenção diária [ 70 ]. Apesar de não haver diferença significativa no acúmulo de placa em relação ao tipo de ligadura [71 , 72 ], o material do braquete também parece influenciar a quantidade e a localização do acúmulo de placa. Os braquetes de aço inoxidável pareceram colher uma quantidade significativamente maior de placa quando comparados aos braquetes de cerâmica, safira e policarbonato. Ao usar braquetes cerâmicos, a maior quantidade de placa se acumulou nas superfícies oclusais e gengivais, enquanto as superfícies mesial e distal acumularam mais placa ao adotar braquetes de aço inoxidável [ 73 - 75 ]. Além disso, as superfícies de aço inoxidável foram 
sugeridas para atrair menos biofilme do que ouro [ 76 - 78] A presença de contenções fixas pode estar associada a um risco de maior acúmulo de placa, recessão gengival e sangramento à sondagem. Pacientes com retentores de fio multitranda exibiram mais acúmulo de placa nas superfícies distais dos dentes anteriores inferiores em comparação com retentores de fio redondos de um único vão [ 79, 80 ].

Para reduzir os riscos de ruptura periodontal durante e após o OTM, mais atenção deve ser dada às características dos aparelhos ortodônticos durante o planejamento. $O$ estado periodontal em pacientes tratados ortodonticamente pode ser avaliado não apenas durante a terapia e após a descolagem, mas provavelmente também durante os acompanhamentos no período de contenção. Em pacientes periodontais submetidos à terapia ortodôntica, o controle da placa deve ser monitorado de perto.

Numerosos artigos discutiram amplamente as vantagens e desvantagens de diferentes tipos de escovas de dente: escovas de dente manuais, sônicas, ortodônticas, elétricas, oscilantes-rotativas, ultrassônicas e iônicas [ 81 - 84 ]. De acordo com a recente atualização de uma revisão Cochrane [ 85 ] baseada em 51 artigos com um total de 4624 participantes, foi sugerido que escovas de dente elétricas podem fornecer um benefício significativo quando comparadas com escovas de dente manuais. Vários estudos em pacientes ortodônticos também apoiaram esses achados e demonstraram maior eficácia das escovas de dente oscilante-rotativas na remoção da placa dentária e redução da gengivite quando comparadas às escovas manuais [ 86,87$]$ Deve-se levar em consideração que a motivação dos pacientes e as instruções repetidas de higiene oral podem ser um fator crucial para pacientes submetidos a OTM com aparelhos fixos [ 88, 89 ]. A motivação de pacientes ortodônticos pode incluir diferentes técnicas educacionais: instruções de higiene oral, mostrando imagens de possíveis complicações, o uso de comprimidos reveladores de placa, demonstrações de técnicas de escovação em modelos e até mesmo mostrando a microscopia de contraste de fase de pacientes de suas amostras de placa [ 90,91$]$

Pacientes ortodônticos que não são capazes de estabelecer níveis satisfatórios de higiene oral devem receber alguns auxiliares adicionais, como vernizes dentais, géis, enxaguatórios bucais ou dentifrícios. A clorexidina ( $\mathrm{CHX}$ ), que pode ser incluída em diferentes tipos de veículos, mostra eficácia antibacteriana contra a inflamação gengival e bactérias cariogênicas e também pode reduzir a gravidade das úlceras traumáticas durante o OTM [ 92 - 96 ]. A discussão sobre os efeitos colaterais relacionados ao uso de longo prazo de $\mathrm{CHX}$, como a coloração dentária, é comumente debatida e considerada relacionada à sua concentração. Ao usar enxaguatórios bucais e dentifrícios com concentrações mais baixas de $\mathrm{CHX}$, é possível reduzir a descoloração do dente sem diferença significativa na redução da formação de placa e inflamação gengival [96 , 97 ]. A inclusão de flúor nos dentifrícios $\mathrm{CHX}$ pode ajudar a fornecer uma melhor profilaxia da 
formação de lesões de manchas brancas, ao mesmo tempo que reduz a inflamação gengival [ 98 ].

A adesão, motivação e manutenção da higiene oral do paciente são universalmente reconhecidas como fatores importantes ao avaliar o impacto do OTM em seu estado periodontal. Esses parâmetros são importantes para a manutenção da condição periodontal após a terapia periodontal não cirúrgica e cirúrgica e devem ser continuados posteriormente. Levando-se em consideração as dificuldades adicionais na higiene dental diária para pacientes ortodônticos durante o tratamento com aparelhos fixos, o monitoramento regular de adultos com predisposição para colapso periodontal durante o OTM é obrigatório. O ortodontista deve estar atento à educação em saúde bucal, com ênfase nas orientações de higiene bucal e cuidados periodontais regulares. Check-ups periodontais e consultas de manutenção de higiene profissional de boa qualidade são essenciais, mesmo após o término do tratamento ortodôntico.

\section{CONCLUSÃO}

O tratamento odontológico multidisciplinar bem coordenado visa fornecer estética, função e prognóstico de longo prazo satisfatórios para os pacientes. Uma cooperação efetiva permite observar os problemas clínicos de diferentes perspectivas e compreender melhor as interações entre as diferentes especialidades. A saúde periodontal é essencial para qualquer forma de tratamento odontológico. Para evitar consequências indesejáveis durante e após o OTM, uma avaliação completa da saúde periodontal deve ser fornecida. Atenção deve ser dada aos parâmetros de higiene dental, especialmente em pacientes que usam aparelhos fixos e em indivíduos periodontalmente suscetíveis.

Nesta revisão, elegemos alguns aspectos clínicos onde os conhecimentos periodontal e ortodôntico se unem para fornecer um quadro mais exaustivo do impacto do tratamento ortodôntico no periodonto. Discutimos os possíveis efeitos do OTM nos tecidos periodontais moles e duros acompanhados pelo uso de aparelhos ortodônticos fixos. Finalmente, a importância da manutenção da saúde, função e estética dos pacientes após a terapia ativa foi enfatizada como uma prioridade no manejo das populações de ambas as especialidades.

Outros campos interessantes onde a interação entre a Ortodontia e a Periodontia é muito importante ainda não foram adequadamente explorados. O momento do tratamento ortodôntico do paciente submetido à terapia periodontal ativa é uma área em que muito poucas evidências foram produzidas. Embora a maioria dos médicos possa 
concordar que o movimento ortodôntico deve ser iniciado após o término da terapia ativa, ainda não existe um protocolo universal que possa ser aplicado a pacientes com dentição periodontal comprometida em tratamento ortodôntico combinado. A influência do protocolo cirúrgico adotado também pode ter um impacto no tempo e as terapias regenerativas podem exigir períodos mais longos de tempo em comparação com os tratamentos periodontais tradicionais, quando uma direção de movimento translúcida é necessária.

Apesar do grande número de artigos publicados, percebemos que faltam boas evidências sobre muitos dos tratamentos, incluindo a ortodontia e a terapia periodontal. Ensaios clínicos bem elaborados avaliando a interação entre essas especialidades aparentemente distantes devem ser incentivados na comunidade odontológica. Avaliar o atendimento ao paciente de apenas um olho da especialidade pode limitar as possibilidades de tratamento quando comparado a uma visão coordenada de cada condição particular. Uma boa perspectiva só pode existir com dois pontos de vista.

\section{THE AUTHORS DECLARE NO CONFLICTS OF INTEREST.}

\section{DECLARAÇÃO CCBY}

Este artigo é uma cópia com adaptação para o português do original "Gorbunkova, Angelina, et al. "Impact of orthodontic treatment on periodontal tissues: a narrative review of multidisciplinary literature." International journal of dentistry 2016 (2016)." 99

Esta cópia com adaptação para o português teve como alteração além do idioma a autoria do artigo.

Os autores da publicação original não deram endosso específico a esta cópia com adaptação para a português, a não ser a licença CCBY 4.0 disponibilizada pelo periódico que publicou o artigo original.

Este é o link da licença: https://creativecommons.org/licenses/by/4.0/

Este é o link do artigo original: https://www.hindawi.com/journals/ijd/2016/4723589/

\section{REFERÊNCIAS}

1. Y. Kim, E. Kwon, Y. Cho, J. Lee, S. Kim, and J. Choi, "Changes in the vertical position of interdental papillae and interseptal bone following the approximation of anterior teeth," The International Journal of Periodontics \& Restorative Dentistry, vol. 34, no. 2, pp. 219-224, 2014.View at: Publisher Site I Google Scholar

2. D. P. Tarnow, A. W. Magner, and P. Fletcher, "The effect of the distance from the contact point to the crest of bone on the presence or absence of the interproximal dental 
papilla," Journal of Periodontology, vol. 63, no. 12, pp. 995-996, 1992.View at: Publisher Site | Google Scholar

3. Y.-J. Wu, Y.-K. Tu, S.-M. Huang, and C.-P. Chan, "The influence of the distance from the contact point to the crest of bone on the presence of the interproximal dental papilla," Chang Gung Medical Journal, vol. 26, no. 11, pp. 822-828, 2003.View at: Google $\underline{\text { Scholar }}$

4. C. Ghezzi, S. Masiero, M. Silvesth, G. Zanotti, and G. Rasperini, "Orthodontic treatment of periodontally involved teeth after tissue regeneration," International Journal of Periodontics \& Restorative Dentistry, vol. 28, no. 6, pp. 559-567, 2008.View at: Google Scholar

5. F. B. Zanatta, T. M. Ardenghi, R. P. Antoniazzi, T. M. P. Pinto, and C. K. Rösing, “Association between gingival bleeding and gingival enlargement and oral health-related quality of life (OHRQoL) of subjects under fixed orthodontic treatment: a cross-sectional study," BMC Oral Health, vol. 12, no. 1, article 53, 2012.View at: $\underline{\text { Publisher Site I Google Scholar }}$

6. H. A. Eid, H. A. M. Assiri, R. Kandyala, R. A. Togoo, and V. S. Turakhia, "Gingival enlargement in different age groups during fixed orthodontic treatment," Journal of International Oral Health, vol. 6, no. 1, pp. 1-4, 2014.View at: Google Scholar

7. J. S. Kloehn and J. S. Pfeifer, "The effect of orthodontic treatment on the periodontium," Angle Orthodontist, vol. 44, no. 2, pp. 127-134, 1974.View at: Google $\underline{\text { Scholar }}$

8. S. Zachrisson and B. U. Zachrisson, "Gingival condition associated with orthodontic treatment," The Angle Orthodontist, vol. 42, no. 1, pp. 26-34, 1972.View at: Google $\underline{\text { Scholar }}$

9. P. Şurlin, A.-M. Rauten, D. Pirici, B. Oprea, L. Mogoantă, and A. Camen, "Collagen IV and MMP-9 expression in hypertrophic gingiva during orthodontic treatment," Romanian Journal of Morphology and Embryology, vol. 53, no. 1, pp. 161-165, 2012.View at: Google $\underline{\text { Scholar }}$

10. U. K. Gursoy, O. Sokucu, V.-J. Uitto et al., "The role of nickel accumulation and epithelial cell proliferation in orthodontic treatment-induced gingival overgrowth," European Journal of Orthodontics, vol. 29, no. 6, pp. 555-558, 2007.View at: Publisher Site I Google $\underline{\text { Scholar }}$

11. C. Marchese, V. Visco, L. Aimati et al., "Nickel-induced keratinocyte proliferation and upmodulation of the keratinocyte growth factor receptor expression," Experimental Dermatology, vol. 12, no. 4, pp. 497-505, 2003.View at: Publisher Site I Google Scholar

12. C. A. Pazzini, L. S. Marques, M. L. Marques, G. O. J. Nior, L. J. Pereira, and S. M. Paiva, 
Impacto do tratamento ortodôntico nos tecidos periodontais: uma revisão narrativa da literatura multidisciplinar

Sá e Ferreira

"Longitudinal assessment of periodontal status in patients with nickel allergy treated with conventional and nickel-free braces," The Angle Orthodontist, vol. 82, no. 4, pp. 653-657, 2012.View at: Publisher Site I Google Scholar

13. C. Maspero, L. Giannini, G. Galbiati, F. Nolet, L. Esposito, and G. Farronato, "Titanium orthodontic appliances for allergic patients," Minerva Stomatologica, vol. 63, no. 11-12, pp. 403-410, 2014.View at: Google Scholar

14. M. C. G. Pantuzo, E. G. Zenóbio, H. D. A. Marigo, and M. A. F. Zenóbio, "Hypersensitivity to conventional and to nickel-free orthodontic brackets," Brazilian Oral Research, vol. 21, no. 4, pp. 298-302, 2007.View at: $\underline{\text { Publisher Site I Google Scholar }}$

15. J. Kurol, A. Ronnerman, and G. Heyden, "Long-term gingival conditions after orthodontic closure of extraction sites. Histological and histochemical studies," European Journal of Orthodontics, vol. 4, no. 2, pp. 87-92, 1982.View at: Publisher Site I Google Scholar

16. P. B. Robertson, L. D. Schultz, and B. M. Levy, "Occurrence and distribution of interdental gingival clefts following orthodontic movement into bicuspid extraction sites," Journal of Periodontology, vol. 48, no. 4, pp. 232-235, 1977.View at: Publisher Site I Google Scholar

17. C. Reichert, L. Gölz, C. Dirk, and A. Jäger, "Retrospective investigation of gingival invaginations: Part I: clinical findings and presentation of a coding system," Journal of Orofacial Orthopedics, vol. 73, no. 4, pp. 307-316, 2012.View at: $\underline{\text { Publisher Site I Google }}$ Scholar

18. A. L. R. Circuns and J. F. C. Tulloch, "Gingival invagination in extraction sites of orthodontic patients: their incidence, effects on periodontal health, and orthodontic treatment," American Journal of Orthodontics, vol. 83, no. 6, pp. 469-476, 1983.View at: $\underline{\text { Publisher Site I Google Scholar }}$

19. L. Gölz, C. Reichert, C. Dirk, and A. Jäger, "Retrospective investigation of gingival invaginations: part II: microbiological findings and genetic risk profile," Journal of Orofacial Orthopedics, vol. 73, no. 5, pp. 387-396, 2012.View at: $\underline{\text { Publisher Site I Google }}$ $\underline{\text { Scholar }}$

20. P. Diedrich and H. Wehrbein, "Orthodontic retraction into recent and healed extraction sites. A histologic study," Journal of Orofacial Orthopedics, vol. 58, no. 2, pp. 90-99, 1997.View at: Google Scholar

21. S. Malkoc, T. Buyukyilmaz, I. Gelgor, and M. Gursel, "Comparison of two different gingivectomy techniques for gingival cleft treatment," The Angle Orthodontist, vol. 74, no. 3, pp. 375-380, 2004.View at: Google Scholar

22. I. N. Ize-Iyamu, B. D. Saheeb, and B. E. Edetanlen, "Comparing the $810 \mathrm{~nm}$ diode laser with conventional surgery in orthodontic soft tissue procedures," Ghana Medical Journal, vol. 
47, no. 3, pp. 107-111, 2013.View at: Google Scholar

23. M. L. B. Pinheiro, T. C. Moreira, and E. J. Feres-Filho, "Guided bone regeneration of a pronounced gingivo-alveolar cleft due to orthodontic space closure," Journal of Periodontology, vol. 77, no. 6, pp. 1091-1095, 2006.View at: Publisher Site I Google Scholar

24. J. Tiefengraber, P. Diedrich, U. Fritz, and P. Lantos, "Orthodontic space closure in combination with membrane supported healing of extraction sockets (MHE). A pilot study," Journal of Orofacial Orthopedics, vol. 63, no. 5, pp. 422-428, 2002.View at: Publisher Site I Google Scholar

25. C. Reichert, M. Wenghöfer, W. Götz, and A. Jäger, "Pilot study on orthodontic space closure after guided bone regeneration," Journal of Orofacial Orthopedics, vol. 72, no. 1, pp. 45-50, 2011.View at: Publisher Site | Google Scholar

26. A. M. Renkema, P. S. Fudalej, A. A. P. Renkema, F. Abbas, E. Bronkhorst, and C. Katsaros, "Gingival labial recessions in orthodontically treated and untreated individuals: a casecontrol study," Journal of Clinical Periodontology, vol. 40, no. 6, pp. 631-637, 2013.View at: Publisher Site I Google Scholar

27. D. Allais and B. Melsen, "Does labial movement of lower incisors influence the level of the gingival margin? A case-control study of adult orthodontic patients," European Journal of Orthodontics, vol. 25, no. 4, pp. 343-352, 2003.View at: $\underline{\text { Publisher Site I Google Scholar }}$

28. G. W. Coatoam, R. G. Behrents, and N. Bissada, "The width of keratinized gingiva during orthodontic treatment: its significance and impact on periodontal status," Journal of Periodontology, vol. 52, no. 6, pp. 307-313, 1981.View at: Publisher Site I Google Scholar

29. A. Andlin-Sobocki and L. Bodin, "Dimensional alterations of the gingiva related to changes of facial/lingual tooth position in permanent anterior teeth of children. A 2-year longitudinal study," Journal of Clinical Periodontology, vol. 20, no. 3, pp. 219-224, 1993.View at: Publisher Site I Google Scholar

30. H. S. Dorfman, "Mucogingival changes resulting from mandibular incisor tooth movement," American Journal of Orthodontics, vol. 74, no. 3, pp. 286-297, 1978.View at: Publisher Site I Google Scholar

31. S. Re, D. Cardaropoli, R. Abundo, and G. Corrente, "Reduction of gingival recession following orthodontic intrusion in periodontally compromised patients," Orthodontics \& Craniofacial Research, vol. 7, no. 1, pp. 35-39, 2004.View at: $\underline{\text { Publisher Site I Google }}$ $\underline{\text { Scholar }}$

32. S. Re, G. Corrente, R. Abundo, and D. Cardaropoli, "Orthodontic treatment in periodontally compromised patients: 12-year report," International Journal of 
Impacto do tratamento ortodôntico nos tecidos periodontais: uma revisão narrativa da literatura multidisciplinar

Sá e Ferreira

Periodontics \& Restorative Dentistry, vol. 20, no. 1, pp. 31-39, 2000.View at: Google $\underline{\text { Scholar }}$

33. G. Djeu, C. Hayes, and S. Zawaideh, "Correlation between mandibular central incisor proclination and gingival recession during fixed appliance therapy," The Angle Orthodontist, vol. 72, no. 3, pp. 238-245, 2002.View at: Google Scholar

34. M. Vehkalahti, "Occurrence of gingival recession in adults," Journal of Periodontology, vol. 60, no. 11, pp. 599-603, 1989.View at: $\underline{\text { Publisher Site I Google Scholar }}$

35. L. Checchi, G. Daprile, M. R. A. Gatto, and G. A. Pelliccioni, "Gingival recession and toothbrushing in an Italian School of Dentistry: a pilot study," Journal of Clinical Periodontology, vol. 26, no. 5, pp. 276-280, 1999.View at: $\underline{\text { Publisher Site I Google Scholar }}$

36. D. C. Matthews, "No good evidence to link toothbrushing trauma to gingival recession," Journal of Evidence-Based Dental Practice, vol. 9, no. 2, article 49, 2008.View at: $\underline{\text { Publisher Site I Google Scholar }}$

37. P. S. Rajapakse, G. I. McCracken, E. Gwynnett, N. D. Steen, A. Guentsch, and P. A. Heasman, "Does tooth brushing influence the development and progression of noninflammatory gingival recession? A systematic review," Journal of Clinical Periodontology, vol. 34, no. 12, pp. 1046-1061, 2007.View at: Publisher Site I Google Scholar

38. J. L. Wennström, "Mucogingival considerations in orthodontic treatment," Seminars in Orthodontics, vol. 2, no. 1, pp. 46-54, 1996.View at: $\underline{\text { Publisher Site I Google Scholar }}$

39. C. Richman, "Is gingival recession a consequence of an orthodontic tooth size and/or tooth position discrepancy? A paradigm shift," Compendium of Continuing Education in Dentistry, vol. 32, no. 4, pp. e73-e79, 2011.View at: Google Scholar

40. Y. A. Mostafa, F. A. El Sharaby, and A. R. El Beialy, "Do alveolar bone defects merit orthodontists' respect?" World Journal of Orthodontics, vol. 10, no. 1, pp. 16-20, 2009.View at: Google Scholar

41. R. Fuhrmann, "Three-dimensional interpretation of periodontal lesions and remodeling during orthodontic treatment. Part III," Journal of Orofacial Orthopedics, vol. 57, no. 4, pp. 224-237, 1996.View at: $\underline{\text { Publisher Site I Google Scholar }}$

42. B. Melsen and D. Allais, "Factors of importance for the development of dehiscences during labial movement of mandibular incisors: a retrospective study of adult orthodontic patients," American Journal of Orthodontics and Dentofacial Orthopedics, vol. 127, no. 5, pp. 552-561, 2005.View at: $\underline{\text { Publisher Site I Google Scholar }}$

43. S. Ruf, K. Hansen, and H. Pancherz, "Does orthodontic proclination of lower incisors in children and adolescents cause gingival recession?" American Journal of Orthodontics and Dentofacial Orthopedics, vol. 114, no. 1, pp. 100-106, 1998.View at: Google Scholar 
44. R. Acunzo, G. Rasperini, P. Cannalire, and G. Farronato, "Influence of periodontal biotype on root surface exposure during orthodontic treatment: a preliminary study," International Journal of Periodontology and Restaurative Dentistry, vol. 35, no. 5, pp. 665-675, 2015.View at: Publisher Site I Google Scholar

45. K. H. Zawawi and M. S. Al-Zahrani, "Gingival biotype in relation to incisors' inclination and position," Saudi Medical Journal, vol. 35, no. 11, pp. 1378-1383, 2014.View at: Google $\underline{\text { Scholar }}$

46. D. R. Cook, B. L. Mealey, R. G. Verrett et al., "Relationship between clinical periodontal biotype and labial plate thickness: an in vivo study," The International Journal of Periodontics \& Restorative Dentistry, vol. 31, no. 4, pp. 345-354, 2011.View at: Google Scholar

47. A. Polson, J. Caton, A. P. Polson, S. Nyman, J. Novak, and B. Reed, "Periodontal response after tooth movement into intrabony defects," Journal of Periodontology, vol. 55, no. 4, pp. 197-202, 1984.View at: $\underline{\text { Publisher Site I Google Scholar }}$

48. C. C. Cirelli, J. A. Cirelli, J. C. D. R. Martins et al., "Orthodontic movement of teeth with intraosseous defects: histologic and histometric study in dogs," American Journal of Orthodontics and Dentofacial Orthopedics, vol. 123, no. 6, pp. 666-675, 2003.View at: Publisher Site I Google Scholar

49. G. Corrente, R. Abundo, S. Re, D. Cardaropoli, and G. Cardaropoli, "Orthodontic movement into infrabony defects in patients with advanced periodontal disease: a clinical and radiological study," Journal of Periodontology, vol. 74, no. 8, pp. 1104-1109, 2003.View at: Publisher Site I Google Scholar

50. D. Cardaropoli, S. Re, G. Corrente, and R. Abundo, "Intrusion of migrated incisors with infrabony defects in adult periodontal patients," American Journal of Orthodontics and Dentofacial Orthopedics, vol. 120, no. 6, pp. 671-677, 2001.View at: $\underline{\text { Publisher }}$ Site I Google Scholar

51. B. Melsen, N. Agerbæk, J. Erikson, and S. Terp, "New attachment through periodontal treatment and orthodontic intrusion," American Journal of Orthodontics and Dentofacial Orthopedics, vol. 94, no. 2, pp. 104-116, 1988.View at: Publisher Site I Google Scholar

52. V. C. da Silva, C. C. Cirelli, F. S. Ribeiro et al., "Intrusion of teeth with class III furcation: a clinical, histologic and histometric study in dogs," Journal of Clinical Periodontology, vol. 35, no. 9, pp. 807-816, 2008.View at: $\underline{\text { Publisher Site | Google Scholar }}$

53. C. C. Cirelli, J. A. Cirelli, J. C. da Rosa Martins et al., "Orthodontic movement of teeth with intraosseous defects: histologic and histometric study in dogs," American Journal of Orthodontics and Dentofacial Orthopedics, vol. 123, no. 6, pp. 666-675, 2003.View at: Publisher Site I Google Scholar 
54. M. Silvestri, G. Rasperini, and S. Milani, " 120 Infrabony defects treated with regenerative therapy: long-term results," Journal of Periodontology, vol. 82, no. 5, pp. 668-675, 2011.View at: Publisher Site | Google Scholar

55. M. A. Reynolds, R. T. Kao, P. M. Camargo et al., "Periodontal regeneration -intrabony defects: a consensus report from the AAP regeneration workshop," Journal of Periodontology, vol. 86, supplement 2, pp. S105-S107, 2015.View at: $\underline{\text { Publisher }}$ Site I Google Scholar

56. R. T. Kao, S. Nares, and M. A. Reynolds, "Periodontal regeneration-intrabony defects: a systematic review from the AAP regeneration workshop," Journal of Periodontology, vol. 86, no. 2, supplement, pp. S77-S104, 2015.View at: Publisher Site | Google Scholar

57. C. E. Nemcovsky, M. Sasson, L. Beny, M. Weinreb, and A. D. Vardimon, "Periodontal healing following orthodontic movement of rat molars with intact versus damaged periodontia towards a bony defect," European Journal of Orthodontics, vol. 29, no. 4, pp. 338-344, 2007.View at: Publisher Site I Google Scholar

58. P. Diedrich, U. Fritz, G. Kinzinger, and J. Angelakis, "Movement of periodontally affected teeth after guided tissue regeneration (GTR)-an experimental pilot study in animals," Journal of Orofacial Orthopedics, vol. 64, no. 3, pp. 214-227, 2003.View at: Google Scholar

59. V. C. da Silva, C. C. Cirelli, F. S. Ribeiro, M. R. Costa, R. C. Comelli Lia, and J. A. Cirelli, "Orthodontic movement after periodontal regeneration of class II furcation: a pilot study in dogs," Journal of Clinical Periodontology, vol. 33, no. 6, pp. 440-448, 2006.View at: $\underline{\text { Publisher Site I Google Scholar }}$

60. M. G. Araújo, D. Carmagnola, T. Berglundh, B. Thilander, and J. Lindhe, "Orthodontic movement in bone defects augmented with Bio-Oss. An experimental study in dogs," Journal of Clinical Periodontology, vol. 28, no. 1, pp. 73-80, 2001.View at: $\underline{\text { Publisher }}$ Site I Google Scholar

61. S. Ogihara and H.-L. Wang, "Periodontal regeneration with or without limited orthodontics for the treatment of 2- or 3-wall infrabony defects," Journal of Periodontology, vol. 81, no. 12, pp. 1734-1742, 2010.View at: $\underline{\text { Publisher Site I Google }}$ $\underline{\text { Scholar }}$

62. M. S. Attia, E. A. Shoreibah, S. A. Ibrahim, and H. A. Nassar, "Histological evaluation of osseous defects combined with orthodontic tooth movement," Journal of the International Academy of Periodontology, vol. 14, no. 1, pp. 7-16, 2012.View at: Google Scholar

63. M. S. Attia, E. A. Shoreibah, S. A. Ibrahim, and H. A. Nassar, "Regenerative therapy of osseous defects combined with orthodontic tooth movement," Journal of the 
Impacto do tratamento ortodôntico nos tecidos periodontais: uma revisão narrativa da literatura multidisciplinar

Sá e Ferreira

International Academy of Periodontology, vol. 14, no. 1, pp. 17-25, 2012.View at: Google $\underline{\text { Scholar }}$

64. R. Rotundo, T. Bassarelli, E. Pace, G. lachetti, J. Mervelt, and G. P. Prato, “Orthodontic treatment of periodontal defects. Part II: a systematic review on human and animal studies," Progress in Orthodontics, vol. 12, no. 1, pp. 45-52, 2011.View at: Publisher Site I Google Scholar

65. K. C. Julien, P. H. Buschang, and P. M. Campbell, "Prevalence of white spot lesion formation during orthodontic treatment," The Angle Orthodontist, vol. 83, no. 4, pp. 641647, 2013.View at: Publisher Site I Google Scholar

66. M. M. C. de Melo, M. G. Cardoso, J. Faber, and A. Sobral, "Risk factors for periodontal changes in adult patients with banded second molars during orthodontic treatment," The Angle Orthodontist, vol. 82, no. 2, pp. 224-228, 2012.View at: $\underline{\text { Publisher Site I Google }}$ $\underline{\text { Scholar }}$

67. I. Ericsson, B. Thilander, J. Lindhe, and H. Okamoto, "The effect of orthodontic tilting movements on the periodontal tissues of infected and non-infected dentitions in dogs," Journal of Clinical Periodontology, vol. 4, no. 4, pp. 278-293, 1977.View at: $\underline{\text { Publisher Site I Google Scholar }}$

68. H. Babacan, O. Sokucu, I. Marakoglu, H. Ozdemir, and R. Nalcaci, "Effect of fixed appliances on oral malodor," American Journal of Orthodontics and Dentofacial Orthopedics, vol. 139, no. 3, pp. 351-355, 2011.View at: Publisher Site I Google Scholar

69. R.-R. Miethke and S. Vogt, "A comparison of the periodontal health of patients during treatment with the Invisalign system and with fixed orthodontic appliances," Journal of Orofacial Orthopedics, vol. 66, no. 3, pp. 219-229, 2005.View at: Publisher Site I Google $\underline{\text { Scholar }}$

70. L. Lombardo, Y. Ö. Ortan, Ö. Gorgun, C. Panza, G. Scuzzo, and G. Siciliani, "Changes in the oral environment after placement of lingual and labial orthodontic appliances," Progress in orthodontics, vol. 14, article 28, 2013.View at: Publisher Site I Google Scholar

71. A. J. Ireland, V. Soro, S. V. Sprague et al., "The effects of different orthodontic appliances upon microbial communities," Orthodontics and Craniofacial Research, vol. 17, no. 2, pp. 115-123, 2014.View at: Publisher Site | Google Scholar

72. W. Sukontapatipark, M. A. El-Agroudi, N. J. Selliseth, K. Thunold, and K. A. Selvig, "Bacterial colonization associated with fixed orthodontic appliances. A scanning electron microscopy study," European Journal of Orthodontics, vol. 23, no. 5, pp. 475-484, 2001.View at: Publisher Site I Google Scholar

73. I. D. Lindel, C. Elter, W. Heuer et al., "Comparative analysis of long-term biofilm formation 
on metal and ceramic brackets," The Angle Orthodontist, vol. 81, no. 5, pp. 907-914, 2011.View at: Publisher Site | Google Scholar

74. T. Eliades, G. Eliades, and W. A. Brantley, "Microbial attachment on orthodontic appliances: I. Wettability and early pellicle formation on bracket materials," American Journal of Orthodontics and Dentofacial Orthopedics, vol. 108, no. 4, pp. 351-360, 1995.View at: Publisher Site I Google Scholar

75. H. F. Saloom, H. S. Mohammed-Salih, and S. F. Rasheed, "The influence of different types of fixed orthodontic appliance on the growth and adherence of microorganisms (in vitro study)," Journal of Clinical and Experimental Dentistry, vol. 5, no. 1, pp. e36-e41, 2013.View at: Publisher Site I Google Scholar

76. M. P. Dittmer, C. F. Hellemann, S. Grade et al., "Comparative three-dimensional analysis of initial biofilm formation on three orthodontic bracket materials," Head \& Face Medicine, vol. 11, article 110, 2015.View at: Publisher Site I Google Scholar

77. M. A. Jongsma, F. D. H. Pelser, H. C. van der Mei et al., "Biofilm formation on stainless steel and gold wires for bonded retainers in vitro and in vivo and their susceptibility to oral antimicrobials," Clinical Oral Investigations, vol. 17, no. 4, pp. 1209-1218, 2013.View at: $\underline{\text { Publisher Site I Google Scholar }}$

78. M. A. Jongsma, H. C. van der Mei, J. Atema-Smit, H. J. Busscher, and Y. Ren, “In vivo biofilm formation on stainless steel bonded retainers during different oral health-care regimens," International Journal of Oral Science, vol. 7, no. 1, pp. 42-48, 2015.View at: Publisher Site I Google Scholar

79. L. Levin, G. R. Samorodnitzky-Naveh, and E. E. Machtei, "The association of orthodontic treatment and fixed retainers with gingival health," Journal of Periodontology, vol. 79, no. 11, pp. 2087-2092, 2008.View at: Publisher Site I Google Scholar

80. K. Al-Nimri, R. Al Habashneh, and M. Obeidat, "Gingival health and relapse tendency: a prospective study of two types of lower fixed retainers," Australian Orthodontic Journal, vol. 25, no. 2, pp. 142-146, 2009.View at: Google Scholar

81. A. Silvestrini Biavati, L. Gastaldo, M. Dessì, F. Silvestrini Biavati, and M. Migliorati, "Manual orthodontic vs. Oscillating-rotating electric toothbrush in orthodontic patients: a randomised clinical trial," European Journal of Paediatric Dentistry, vol. 11, no. 1, pp. 200202, 2010.View at: Google Scholar

82. M. R. Costa, V. C. Silva, M. N. Miqui, T. Sakima, D. M. P. Spolidorio, and J. A. Cirelli, "Efficacy of ultrasonic, electric and manual toothbrushes in patients with fixed orthodontic appliances," The Angle Orthodontist, vol. 77, no. 2, pp. 361-366, 2007.View at: Publisher Site I Google Scholar 
83. A. Borutta, E. Pala, and T. Fischer, "Effectiveness of a powered toothbrush compared with a manual toothbrush for orthodontic patients with fixed appliances," Journal of Clinical Dentistry, vol. 13, no. 4, pp. 131-137, 2002.View at: Google Scholar

84. J. Hickman, D. T. Millett, L. Sander, E. Brown, and J. Love, "Powered vs manual tooth brushing in fixed appliance patients: a short term randomized clinical trial," The Angle Orthodontist, vol. 72, no. 2, pp. 135-140, 2002.View at: Google Scholar

85. M. Yaacob, H. V. Worthington, S. A. Deacon et al., "Powered versus manual toothbrushing for oral health," Cochrane Database of Systematic Reviews, no. 6, Article ID CD002281, 2014.View at: Google Scholar

86. C. Erbe, M. Klukowska, I. Tsaknaki, H. Timm, J. Grender, and H. Wehrbein, "Efficacy of 3 toothbrush treatments on plaque removal in orthodontic patients assessed with digital plaque imaging: a randomized controlled trial," American Journal of Orthodontics and Dentofacial Orthopedics, vol. 143, no. 6, pp. 760-766, 2013.View at: $\underline{\text { Publisher }}$ Site I Google Scholar

87. A. Silvestrini Biavati, L. Gastaldo, M. Dessì, F. Silvestrini Biavati, and M. Migliorati, "Manual orthodontic vs. oscillating-rotating electric toothbrush in orthodontic patients: a randomised clinical trial," European Journal of Paediatric Dentistry, vol. 11, no. 1, pp. 200202, 2010.View at: Google Scholar

88. C. Kossack and P.-G. Jost-Brinkmann, "Plaque and gingivitis reduction in patients undergoing orthodontic treatment with fixed appliances-comparison of toothbrushes and interdental cleaning aids. A 6-month clinical single-blind trial," Journal of Orofacial Orthopedics, vol. 66, no. 1, pp. 20-38, 2005.View at: $\underline{\text { Publisher Site I Google Scholar }}$

89. I. Marini, F. Bortolotti, S. Incerti Parenti, M. R. Gatto, and G. Alessandri Bonetti, "Combined effects of repeated oral hygiene motivation and type of toothbrush on orthodontic patients: a blind randomized clinical trial," The Angle Orthodontist, vol. 84, no. 5, pp. 896-901, 2014.View at: Publisher Site | Google Scholar

90. S. Acharya, A. Goyal, A. K. Utreja, and U. Mohanty, "Effect of three different motivational techniques on oral hygiene and gingival health of patients undergoing multibracketed orthodontics," The Angle Orthodontist, vol. 81, no. 5, pp. 884-888, 2011.View at: Publisher Site I Google Scholar

91. Y. Peng, R. Wu, W. Qu et al., "Effect of visual method vs plaque disclosure in enhancing oral hygiene in adolescents and young adults: a single-blind randomized controlled trial," American Journal of Orthodontics and Dentofacial Orthopedics, vol. 145, no. 3, pp. 280-286, 2014.View at: Publisher Site I Google Scholar

92. G. B. Anderson, J. Bowden, E. C. Morrison, and R. G. Caffesse, "Clinical effects of chlorhexidine mouthwashes on patients undergoing orthodontic treatment," American 
Journal of Orthodontics and Dentofacial Orthopedics, vol. 111, no. 6, pp. 606-612, 1997.View at: Google Scholar

93. K. W. Albertsson, A. Persson, and J. W. V. van Dijken, "Effect of essential oils containing and alcohol-free chlorhexidine mouthrinses on cariogenic micro-organisms in human saliva," Acta Odontologica Scandinavica, vol. 71, no. 3-4, pp. 883-891, 2013.View at: Publisher Site I Google Scholar

94. R. Attin, E. Yetkiner, A. Aykut-Yetkiner, M. Knösel, and T. Attin, "Effect of chlorhexidine varnish application on Streptoococcus mutans colonisation in adolescents with fixed orthodontic appliances," Australian Orthodontic Journal, vol. 29, no. 1, pp. 52-57, 2013.View at: Google Scholar

95. O. Baygin, T. Tuzuner, M.-B. Ozel, and O. Bostanoglu, "Comparison of combined application treatment with one-visit varnish treatments in an orthodontic population," Medicina Oral, Patologia Oral y Cirugia Bucal, vol. 18, no. 2, pp. e362-e370, 2013.View at: Publisher Site I Google Scholar

96. P. V. P. Oltramari-Navarro, J. M. Titarelli, J. A. Marsicano et al., "Effectiveness of $0.50 \%$ and $0.75 \%$ chlorhexidine dentifrices in orthodontic patients: a double-blind and randomized controlled trial," American Journal of Orthodontics and Dentofacial Orthopedics, vol. 136, no. 5, pp. 651-656, 2009.View at: Publisher Site I Google Scholar

97. C.-P. Ernst, K. Prockl, and B. Willershausen, "The effectiveness and side effects of $0.1 \%$ and $0.2 \%$ chlorhexidine mouthrinses: a clinical study," Quintessence International, vol. 29, no. 7, pp. 443-448, 1998.View at: Google Scholar

98. K. P. K. Olympio, P. A. P. Bardal, J. R. D. M. Bastos, and M. A. R. Buzalaf, "Effectiveness of a chlorhexidine dentifrice in orthodontic patients: a randomized-controlled trial," Journal of Clinical Periodontology, vol. 33, no. 6, pp. 421-426, 2006.View at: Publisher Site I Google Scholar

99. Gorbunkova, Angelina, et al. "Impact of orthodontic treatment on periodontal tissues: a narrative review of multidisciplinary literature." International journal of dentistry 2016 (2016). 